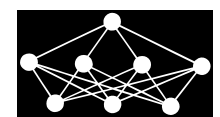

\title{
FLOPPY LOGIC AS A GENERALIZATION OF STANDARD BOOLEAN LOGIC
}

\author{
P. Provinsky*
}

\begin{abstract}
The topic of this article is a floppy logic, a new multi-valued logic. Floppy logic is related to fuzzy logic and the theory of probability, but it also has interesting links to probability logic and standard Boolean logic. It provides a consistent and simple theory that is easy to apply in practice.

This article examines the isomorphism theorem, which plays an important role in floppy logic. The theorem is described and proved.

The most important consequences of the isomorphism theorem are:

1) All statements which are equivalent in standard Boolean logic are also equivalent in floppy logic.

2) Floppy logic has all the properties of standard Boolean logic which can be formulated as an equivalence. These include, for example, distributivity, the contradiction law, the law of excluded middle, and others.

The article mainly examines floppy implication. We show that floppy implication does not satisfy Adam's Thesis and that floppy logic is not limited by Lewis' triviality result.

We also present a range of inference rules which are generalizations of modus ponens and modus tollens. These rules hold in floppy logic, and of course, also apply to standard Boolean logic.

All these results lead us to the notion that floppy logic is a many-valued generalization of standard Boolean logic.

Key words: floppy logic, fuzzy logic, multi-valued logic, probabilistic logic, rules of inference, modus ponens, modus tollens
\end{abstract}

Received: January 21, 2020

DOI: $10.14311 / N N W .2020 .30 .014$

Revised and accepted: June 30, 2020

\section{Introduction}

Multi-valued logic is an important component of modern technologies. It can be found, for example, in home appliances, autonomous vehicles [6] and automated traffic management [3].

The first article discussing multi-valued logic, written by Jan Łukasiewicz, appeared in 1920 [5].

The present paper focuses on infinite-valued logic, which holds that a truth value can be any real number from the interval $[0,1]$. This property has two wellknown logics: probabilistic logic and fuzzy logic.

\footnotetext{
*Pavel Provinský; Czech Technical University in Prague, Faculty of Transportation Sciences, Na Florenci 25, Praha 110 00, Czech Republic, E-mail: provipav@fd.cvut.cz
} 


\subsection{Probabilistic Logic}

The best-known works on probabilistic logic were written by Robert C. Stalnaker in 1970 [10] and Ernest Wilcox Adams in 1975 [1].

In these theories, probability is a generalization of the truth value. The main hypothesis behind these theories is the so-called Adams' Thesis, which states that the probabilities of implications are equal to conditional probabilities:

$$
P(A \Rightarrow B)=P(B \mid A), \quad \text { if } P(A)>0 .
$$

Adams adds that:

$$
P(A \Rightarrow B)=1, \quad \text { if } P(A)=0 .
$$

David Lewis showed [4] that probability logic has one major problem: it cannot be used to work with sentences wherein an implication contains some other implication. For example, sentence $(A \wedge(A \Rightarrow B)) \Rightarrow B$ departs from the possibilities of probabilistic logic. This outcome is known as the Lewis' triviality result.

\section{$1.2 \quad$ Fuzzy Logic}

The first article about fuzzy logic was written by Lotfali Askar Zadeh in 1965 [11]. In that work, the author introduced fuzzy sets and their membership functions. The membership function of a fuzzy set can obtain any value from the interval $[0,1]$. Therefore, an element can belong to a fuzzy set, for example, only to $20 \%$.

Zadeh suggested two methods of operating with fuzzy sets. From these two methods, Gödel and product fuzzy logics were devised. The following years saw the invention of Łukasiewicz, drastic, and many other fuzzy logics. Fuzzy logic has since become popular and the most used multi-value logic.

However, fuzzy logic also has its problems. The different types of fuzzy logic provide different results. It is not clear which fuzzy logic should be used in particular situations.

The second problem is that no fuzzy logic meets all of the laws of the standard two-valued logic. The proof of this fact can be found, for example, in [7]. Details about which laws are met in the most commonly used fuzzy logics are given in Tab. I.

\subsection{Questions}

Three interesting questions arise from the previous sentences about probabilistic logic and fuzzy logic:

1) Does an infinite-valued logic that preserves all the laws of standard binary logic exist?

2) Does a logic that works with sentence probabilities and avoids Lewis' triviality result exist?

3) Probabilistic logic works with probabilities, while fuzzy logic works with membership functions. Which method, seen from the perspective of the future, is the most suitable? 
Provinský P.: Floppy logic as a generalization of standard Boolean logic

\begin{tabular}{lcccc}
\hline & & G & Ł & P \\
\hline involution & $\neg(\neg A)=A$ & OK & OK & OK \\
\hline commutativity & $A \vee B=B \vee A$ & OK & OK & OK \\
& $A \wedge B=B \wedge A$ & & & \\
\hline associativity & $(A \vee B) \vee C=A \vee(B \vee C)$ & OK & OK & OK \\
& $(A \wedge B) \wedge C=A \wedge(B \wedge C)$ & & & \\
\hline distributivity & $A \wedge(B \vee C)=(A \wedge B) \vee(A \wedge C)$ & OK & $\times$ & $\times$ \\
& $A \vee(B \wedge C)=(A \vee B) \wedge(A \vee C)$ & & & \\
\hline idempotence & $A \vee A=A$ & OK & $\times$ & $\times$ \\
& $A \wedge A=A$ & & & \\
\hline absorption & $A \vee(A \wedge B)=A$ & OK & $\times$ & $\times$ \\
& $A \wedge(A \vee B)=A$ & & & \\
\hline absorption with 1 and 0 & $A \vee 1=1$ & OK & OK & OK \\
& $A \wedge 0=0$ & & & \\
\hline neutral elements & $A \vee 0=A$ & OK & OK & OK \\
& $A \wedge 1=A$ & & & \\
\hline law of contradiction & $A \wedge \neg A=0$ & $\times$ & OK & $\times$ \\
\hline law of excluded middle & $A \vee \neg A=1$ & $\times$ & OK & $\times$ \\
\hline De Morgan's laws & $\neg(A \vee B)=\neg A \wedge \neg B$ & OK & OK & OK \\
\hline & $\neg(A \wedge B)=\neg A \vee \neg B$ & & & \\
\hline Tab & & & &
\end{tabular}

Tab. I Logical laws in the most commonly used fuzzy logics. $G=$ Gödel logic, $E=$ Eukasiewicz logic, $P=$ product logic, all with the standard negation. Data are drawn from [r].

\subsection{Floppy Logic}

In September 2014, Ivan Nagy, my colleague, posed an interesting question of whether it would be possible somehow to link statistics and fuzzy logic consistently. It would be very useful. Frequently, more complex systems described with fuzzy logic simultaneously need statistical data about this system to be processed.

Ivan Nagy immediately suggested a solution: if a structure that met all the Kolmogorov Axioms of probability theory was found in the world of fuzzy sets, then all probability tools could be consistently used when working with fuzzy sets. This idea was immediately interesting, and after two weeks, the first results were compiled.

The first article about this new logic was published in 2017 [8]. In that paper, a theoretical foundation was given. This new logic was named floppy logic.

The second article [9], published in 2018, gave many examples of how to work with floppy logic. 


\section{A brief overview of floppy logic}

\subsection{Introduction of floppy logic}

Let $A_{1}, A_{2}, \ldots$ be primary fuzzy sets.

Let $\mu_{A_{1}}(x), \mu_{A_{2}}(x), \ldots$ be the membership functions of fuzzy sets $A_{1}, A_{2}, \ldots$

Let $X$ be the domain of the membership functions $\mu_{A_{1}}(x), \mu_{A_{2}}(x), \ldots$

Let $\boldsymbol{S}$ be the set of all primary fuzzy sets $A_{1}, A_{2}, \ldots$

Let $\mathcal{P}(\boldsymbol{S})$ be the power set of $\boldsymbol{S}$.

Let $\boldsymbol{S}_{\boldsymbol{x}}$ be a set of all primary fuzzy sets $A_{i}$ whose membership functions are greater than 0 for given $x$.

Let the following assumptions be met:

Assumption 1. For all $x \in X, \boldsymbol{S}_{\boldsymbol{x}}$ is a finite or countable set.

Assumption 2. Membership functions of fuzzy sets $A_{i} \in \boldsymbol{S}$ assume values from the interval $[0,1]$.

Assumption 3.

$$
\forall x \in X: \sum_{A_{i} \in S} \mu_{A_{i}}(x)=1 .
$$

Assumption 4. A measure space $(X, \mathcal{A}, P)$ is defined on the set $X$, where $\mathcal{A}$ is a $\sigma$-algebra on $X$, and $P$ is a probability measure.

Assumption 5. All membership functions $\mu_{A_{i}}(x)$ of fuzzy sets $A_{i} \in \boldsymbol{S}$ are measurable on the sets $X_{i} \in \mathcal{A}$ corresponding to measure $P$.

Definition 1. All subsets of $\boldsymbol{S}$ are called floppy sets. Floppy sets are denoted in bold capital letters.

Definition 2. To each floppy set $\boldsymbol{B} \subseteq \boldsymbol{S}$, a function $\boldsymbol{\mu}_{\boldsymbol{B}}(x)$ is associated. The function $\boldsymbol{\mu}_{\boldsymbol{B}}(x)$ is defined as:

$$
\boldsymbol{\mu}_{\boldsymbol{B}}(x)=\sum_{A_{i} \in \boldsymbol{B}} \mu_{A_{i}}(x) .
$$

The function $\boldsymbol{\mu}_{\boldsymbol{B}}(x)$ is called the cumulative membership function of the floppy set and is denoted by bold Greek letter $\boldsymbol{\mu}$.

Definition 3. To each floppy set $\boldsymbol{B} \subseteq \boldsymbol{S}$, a number $R(\boldsymbol{B})$ is associated. The number $R(\boldsymbol{B})$ is defined as:

$$
R(\boldsymbol{B})=\int_{X} \boldsymbol{\mu}_{\boldsymbol{B}}(x) \mathrm{d} P,
$$

where this integral is the Lebesgue integral and $P$ is the probability measure given in Assumption 4. The number $R(\boldsymbol{B})$ is called the probability of the floppy set and is denoted by capital letter $R$.

Definition 4. The space $(\boldsymbol{S}, \mathcal{P}(\boldsymbol{S}), R)$ is called a basic floppy probability space.

In floppy logic, the following theorem applies:

Theorem 1. Each basic floppy probability space satisfies all Kolmogorov Axioms.

This theorem is proved in [8]. 


\section{$2.2 \quad$ Remarks}

Remark 1. By proving Theorem 1, the objective set by Ivan Nagy is achieved. A structure that meets all Kolmogorov Axioms is found in the world of fuzzy sets. Thus, all the standard probabilistic tools and concepts in floppy logic can be used. For example, Bayes' theorem can be applied, or the mean value or median of the floppy set can be introduced consistently.

Remark 2. The result of Theorem 1 could be thought of as trivial since the existence of a probability space is assumed in Assumption 4 and the existence of a probability space is proved in Theorem 1 . This is not true because $(X, \mathcal{A}, P)$ and $(\boldsymbol{S}, \mathcal{P}(\boldsymbol{S}), R)$ are completely different probability spaces. The probability measures $P$ and $R$ were distinguished for this reason.

Remark 3. The floppy set is crisp set of the primary fuzzy sets. Its cumulative membership function is the sum of membership functions of its elements.

Remark 4. In the previous articles [8] and [9], the function $\boldsymbol{\mu}_{\boldsymbol{B}}(x)$ was called the membership function of the floppy set. This denomination was chosen to indicate that this function has a very similar role in floppy logic as the membership function of the fuzzy set in fuzzy logic. However, this denomination led to misunderstandings, therefore it was changed.

Remark 5. The intersection of two different one-element floppy sets is equal to an empty set. Thus, when a certain reality is modeled, the primary fuzzy sets that respect this property must be selected.

For example, the temperature of water can be described with the following three primary fuzzy sets: unpleasantly cold, pleasant, and unpleasantly warm. No one would ever suggest that water is, for example, unpleasantly cold and pleasant simultaneously. Therefore, it is acceptable that the intersection of these two properties (one-element floppy sets) is an empty set.

On the other hand, the following primary fuzzy sets cannot be used: cold, pleasant, and warm, since water can be, for example, warm and pleasant simultaneously.

Remark 6. In the previous articles [8] and [9], Assumption 1 was stronger: $\boldsymbol{S}$ is a finite or countable set. However, this stronger version is not necessary in order to prove Theorem 1, therefore this assumption was changed.

Remark 7. Floppy logic is not truth-functional. It means that $\boldsymbol{\mu}_{\boldsymbol{A} \cap \boldsymbol{B}}(x)$ cannot be computed from $\boldsymbol{\mu}_{\boldsymbol{A}}(x)$ and $\boldsymbol{\mu}_{\boldsymbol{B}}(x)$. The common elements (primary fuzzy sets) of floppy sets $\boldsymbol{A}$ and $\boldsymbol{B}$ must be known.

Similarly, in probability theory, the probability of an event $A \cap B$ cannot be calculated from the probabilities of events $A$ and $B$.

On the other hand, standard binary logic and fuzzy logics are truth-functional.

Remark 8. Many examples of how to work with floppy sets in practice are presented in [9]. 


\section{Isomorphism theorem}

\subsection{Formulation}

The present paper now introduces a very important theorem in floppy logic. A similar statement appeared in [8], but no detailed mathematical proof has yet been provided.

Theorem 2. Let $\varepsilon=(E, \wedge, \vee, \neg, \perp, \top$, 三) be a finite Boolean algebra of sentences.

Let $E_{A}=\left\{U_{1}, U_{2}, U_{3}, \ldots U_{n}\right\}$ be a set of all atoms of this Boolean algebra and $n$ number of these atoms.

Let $\boldsymbol{S}=\left\{A_{1}, A_{2}, A_{3}, \ldots A_{n}\right\}$ be a set of $n$ primary fuzzy sets and $X$ be their domain.

Let $\delta=\left(\mathcal{P}(\boldsymbol{S}), \cap, \cup,^{\prime}, \emptyset, \boldsymbol{S},=\right)$ be a Boolean algebra of subsets of $\boldsymbol{S}$.

Let $f$ be a relation that meets:

$$
\begin{array}{ll}
\text { a. } & \forall k \in\{1,2,3, \ldots n\}: f\left(U_{k}\right)=\left\{A_{k}\right\}, \\
\text { b. } & \forall V, W \in E: f(V \wedge W)=f(V) \cap f(W), \\
\text { c. } & \forall V, W \in E: f(V \vee W)=f(V) \cup f(W), \\
\text { d. } & \forall V \in E: f(\neg V)=f(V)^{\prime} .
\end{array}
$$

Then:

1. $f$ is an isomorphism,

2. $\varepsilon$ and $\delta$ are isomorphic,

3. $\forall V, W \in E: V \equiv W \Leftrightarrow f(V)=f(W)$,

4. $\forall V, W \in E, \forall x \in X: V \equiv W \Rightarrow \boldsymbol{\mu}_{f(V)}(x)=\boldsymbol{\mu}_{f(W)}(x)$

5. $\forall V, W \in E: V \equiv W \Rightarrow R(f(V))=R(f(W))$.

The terms Boolean algebra, atom, and isomorphism are explained in Appendix I. Theorem 2 is proved in Appendix II.

\subsection{Consequences}

Consequence 1. Theorem 2 states that sentences that are equivalent in standard Boolean logic (and consisting of a finite set of atomic statements) are assigned the same floppy set, and hence also the same cumulative membership function, and thus also the same probability. This means that statements which are equivalent in standard Boolean logic are also equivalent in floppy logic.

Consequence 2. It means that floppy logic meets all the standard binary logic properties that are formulated as an equivalence of two sentences. All the properties listed in Tab. I are thus, especially, satisfied.

Consequence 3. Floppy logic can hence be considered a generalization of standard Boolean logic.

Consequence 4. Standard Boolean logic often models statements using sets, such as Venn diagrams. In view of Theorem 2, these sets can be interpreted as floppy sets. 
Consequence 5. Theorem 2 states that Boolean algebras $\varepsilon$ and $\delta$ are isomorphic. Thus, if it is appropriate, logical and set operations can be used together or exchanged.

Consequence 6. The probability of implication, for example, can be expressed as follows:

$$
R(\boldsymbol{A} \Rightarrow \boldsymbol{B})=R(\neg \boldsymbol{A} \vee(\boldsymbol{A} \wedge \boldsymbol{B}))=R\left(\boldsymbol{A}^{\prime} \cup(\boldsymbol{A} \cap \boldsymbol{B})\right) .
$$

$\boldsymbol{A}^{\prime}$ and $\boldsymbol{A} \cap \boldsymbol{B}$ are disjunct sets, thus:

$$
R\left(\boldsymbol{A}^{\prime} \cup(\boldsymbol{A} \cap \boldsymbol{B})\right)=R\left(\boldsymbol{A}^{\prime}\right)+R(\boldsymbol{A} \cap \boldsymbol{B}) .
$$

Theorem 1 declares that $R$ fulfills all the properties of probability. Standard formulas for $R(\boldsymbol{A} \cap \boldsymbol{B})$ and $R\left(\boldsymbol{A}^{\prime}\right)$ can thus be used, obtaining:

$$
R(\boldsymbol{A} \Rightarrow \boldsymbol{B})=1-R(\boldsymbol{A})+R(\boldsymbol{B} \mid \boldsymbol{A}) \cdot R(\boldsymbol{A}) .
$$

It was found that Adams' Thesis in floppy logic does not apply.

A comparison of the probability of implication in probabilistic logic and floppy logic is shown in Fig. 1.

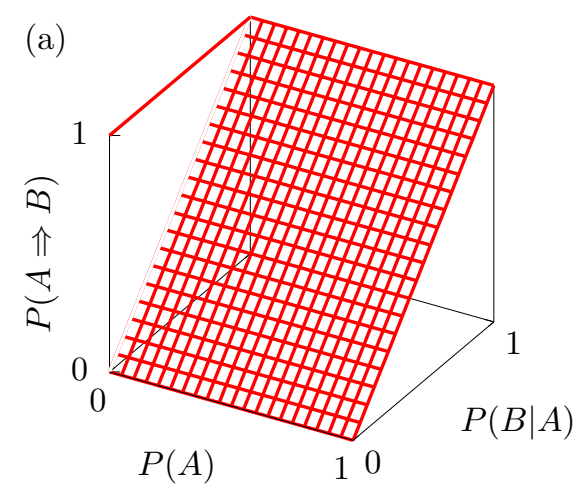

Adams' Thesis

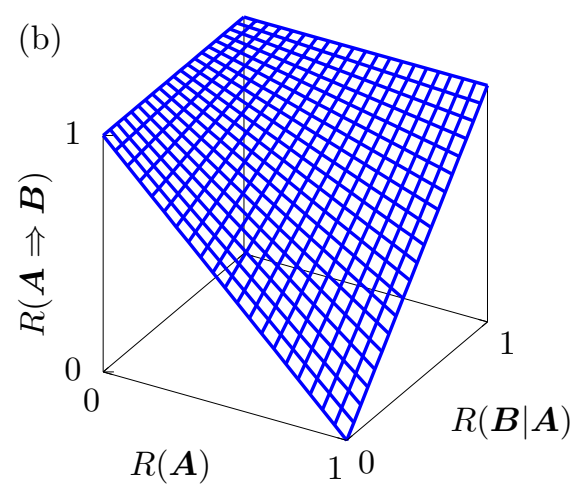

Floppy implication

Fig. 1 Comparison of the probability of implication in probabilistic logic, according to Adams' Thesis (a), and floppy logic (b).

Consequence 7. Similarly, transitivity of implication can be expressed as follows:

$$
R[[(\boldsymbol{A} \Rightarrow \boldsymbol{B}) \wedge(\boldsymbol{B} \Rightarrow \boldsymbol{C})] \Rightarrow[\boldsymbol{A} \Rightarrow \boldsymbol{C}]]=1 .
$$

Floppy logic is not limited by Lewis' triviality result.

Consequence 8. In floppy logic, both probabilities (as in probabilistic logic) and cumulative membership functions (similarly as in fuzzy logic) can be worked with. 
Sentences equivalent in standard binary logic have the same cumulative membership functions and probabilities. It is very surprising that both of these different generalizations of the truth-value meet each other in a single theory.

As is shown in [8], the relationship between probability and the cumulative membership function is, in floppy logic, given by the formula:

$$
\boldsymbol{\mu}_{\boldsymbol{A}}(x)=R(\boldsymbol{A} \mid x) .
$$

\section{A noteworthy implication}

One of the important consequences of the isomorphism theorem is the formula for floppy implication (15).

This formula, along with the law of total probability and Bayes' theorem, allows us to infer a range of interesting relationships for floppy implications. These relationships hold not only for floppy logic but also for standard Boolean logic, assuming that we replace the probabilities of sentences (floppy sets) with truth values 0 or 1 .

\subsection{Bayes' theorem}

$$
\begin{aligned}
R(\boldsymbol{B} \mid \boldsymbol{A}) & =\frac{R(\boldsymbol{A} \mid \boldsymbol{B}) \cdot R(\boldsymbol{B})}{R(\boldsymbol{A})}, \\
R(\boldsymbol{B} \mid \boldsymbol{A}) \cdot R(\boldsymbol{A}) & =R(\boldsymbol{A} \mid \boldsymbol{B}) \cdot R(\boldsymbol{B}), \\
R(\boldsymbol{A} \Rightarrow \boldsymbol{B})-1+R(\boldsymbol{A}) & =R(\boldsymbol{B} \Rightarrow \boldsymbol{A})-1+R(\boldsymbol{B}), \\
R(\boldsymbol{B} \Rightarrow \boldsymbol{A})-R(\boldsymbol{A} \Rightarrow \boldsymbol{B}) & =R(\boldsymbol{A})-R(\boldsymbol{B}), \\
R(\boldsymbol{B} \Rightarrow \boldsymbol{A}) & =R(\boldsymbol{A} \Rightarrow \boldsymbol{B})+R(\boldsymbol{A})-R(\boldsymbol{B}) .
\end{aligned}
$$

And here is a variant for cumulative membership function:

$$
\boldsymbol{\mu}_{\boldsymbol{B} \Rightarrow \boldsymbol{A}}(x)=\boldsymbol{\mu}_{\boldsymbol{A} \Rightarrow \boldsymbol{B}}(x)+\boldsymbol{\mu}_{\boldsymbol{A}}(x)-\boldsymbol{\mu}_{\boldsymbol{B}}(x) .
$$

\subsection{Contraposition of implication}

A contraposition of implication is a logical law that states that implication $A \Rightarrow B$ is logically equivalent to implication $\neg B \Rightarrow \neg A$.

In floppy logic, the law of contraposition follows directly from Theorem 2 . The variants for probabilities and cumulative membership functions are provided below:

$$
\begin{aligned}
R(\boldsymbol{A} \Rightarrow \boldsymbol{B}) & =R(\neg \boldsymbol{B} \Rightarrow \neg \boldsymbol{A}), \\
\boldsymbol{\mu}_{\boldsymbol{A} \Rightarrow \boldsymbol{B}}(x) & =\boldsymbol{\mu}_{\neg \boldsymbol{B} \Rightarrow \neg \boldsymbol{A}}(x) .
\end{aligned}
$$

\subsection{Modus ponens}

Modus ponens is a logical rule that says: If $A$ holds and $A \Rightarrow B$ holds, then $B$ holds. 
A generalization of modus ponens in floppy logic could look as follows: We know the probability of $\boldsymbol{A}$ and the probability of $\boldsymbol{A} \Rightarrow \boldsymbol{B}$. What is the probability of $\boldsymbol{B}$ ?

The correct answer is that we do not have enough information to answer the question.

Let us now consider the case where we are given more information: for instance, we may know the probability of $\neg \boldsymbol{A} \Rightarrow \boldsymbol{B}$. We can then write:

$$
\begin{aligned}
R(\boldsymbol{B}) & =R(\boldsymbol{A} \cap \boldsymbol{B})+R(\neg \boldsymbol{A} \cap \boldsymbol{B})= \\
& =R(\boldsymbol{B} \mid \boldsymbol{A}) \cdot R(\boldsymbol{A})+R(\boldsymbol{B} \mid \neg \boldsymbol{A}) \cdot R(\neg \boldsymbol{A})= \\
& =R(\boldsymbol{A} \Rightarrow \boldsymbol{B})-1+R(\boldsymbol{A})+R(\neg \boldsymbol{A} \Rightarrow \boldsymbol{B})-1+R(\neg \boldsymbol{A}), \\
R(\boldsymbol{B}) & =R(\boldsymbol{A} \Rightarrow \boldsymbol{B})+R(\neg \boldsymbol{A} \Rightarrow \boldsymbol{B})-1 .
\end{aligned}
$$

This is a very interesting generalization of modus ponens. It is surprising that we do not need $R(\boldsymbol{A})$ to compute $R(\boldsymbol{B})$. All the required information about $\boldsymbol{A}$ is given in the probabilities $R(\boldsymbol{A} \Rightarrow \boldsymbol{B})$ and $R(\neg \boldsymbol{A} \Rightarrow \boldsymbol{B})$.

Let us now show that the equation (29) is indeed a generalization of modus ponens: If $R(\boldsymbol{A})=1$ and $R(\boldsymbol{A} \Rightarrow \boldsymbol{B})=1$, then:

$$
R(\neg \boldsymbol{A})=0, \quad R(\neg \boldsymbol{A} \wedge \boldsymbol{B})=0, \quad R(\neg \boldsymbol{A} \Rightarrow \boldsymbol{B})=1, \quad R(\boldsymbol{B})=1 .
$$

Other generalizations of modus ponens can be derived similarly:

$$
\begin{aligned}
& R(\boldsymbol{B})=R(\boldsymbol{A} \Rightarrow \boldsymbol{B})+R(\boldsymbol{A})-R(\boldsymbol{B} \Rightarrow \boldsymbol{A}), \\
& R(\boldsymbol{B})=2-R(\boldsymbol{B} \Rightarrow \neg \boldsymbol{A})-R(\boldsymbol{B} \Rightarrow \boldsymbol{A}), \\
& R(\boldsymbol{B})=R(\neg \boldsymbol{A} \Rightarrow \boldsymbol{B})+R(\neg \boldsymbol{A})-R(\boldsymbol{B} \Rightarrow \neg \boldsymbol{A}) .
\end{aligned}
$$

Variants for cumulative membership functions are here:

$$
\begin{aligned}
& \boldsymbol{\mu}_{B}(x)=\boldsymbol{\mu}_{\boldsymbol{A} \Rightarrow \boldsymbol{B}}(x)+\boldsymbol{\mu}_{\neg A \Rightarrow B}(x)-1, \\
& \boldsymbol{\mu}_{B}(x)=\boldsymbol{\mu}_{A \Rightarrow B}(x)+\boldsymbol{\mu}_{\boldsymbol{A}}(x)-\boldsymbol{\mu}_{B \Rightarrow \boldsymbol{A}}(x), \\
& \boldsymbol{\mu}_{B}(x)=2-\boldsymbol{\mu}_{B \Rightarrow \neg \boldsymbol{A}}(x)-\boldsymbol{\mu}_{B \Rightarrow \boldsymbol{A}}(x), \\
& \boldsymbol{\mu}_{\boldsymbol{B}}(x)=\boldsymbol{\mu}_{\neg \boldsymbol{A} \Rightarrow \boldsymbol{B}}(x)+\boldsymbol{\mu}_{\neg \boldsymbol{A}}(x)-\boldsymbol{\mu}_{B \Rightarrow \neg \boldsymbol{A}}(x) .
\end{aligned}
$$

\subsection{Modus ponens for a finite partition of the sample space}

Let $\left\{\boldsymbol{A}_{i}\right\}$ be a finite set of $n$ pairwise disjoint floppy sets whose union is the whole sample space. We can then generalize modus ponens as follows:

$$
\begin{aligned}
& R(\boldsymbol{B})=\sum_{i=1}^{n} R\left(\boldsymbol{A}_{i} \Rightarrow \boldsymbol{B}\right)-n+1, \\
& R(\boldsymbol{B})=\frac{n-\sum_{i=1}^{n} R\left(\boldsymbol{B} \Rightarrow \boldsymbol{A}_{i}\right)}{n-1},
\end{aligned}
$$




$$
\begin{aligned}
R(B) & =n-\sum_{i=1}^{n} R\left(\boldsymbol{B} \Rightarrow \neg \boldsymbol{A}_{i}\right), \\
R(\boldsymbol{B}) & =\frac{\sum_{i=1}^{n} R\left(\neg \boldsymbol{A}_{i} \Rightarrow \boldsymbol{B}\right)-1}{n-1}, \\
\boldsymbol{\mu}_{\boldsymbol{B}}(x) & =\sum_{i=1}^{n} \boldsymbol{\mu}_{\boldsymbol{A}_{i} \Rightarrow \boldsymbol{B}}(x)-n+1, \\
\boldsymbol{\mu}_{\boldsymbol{B}}(x) & =\frac{n-\sum_{i=1}^{n} \boldsymbol{\mu}_{\boldsymbol{B} \Rightarrow \boldsymbol{A}_{i}}(x)}{n-1}, \\
\boldsymbol{\mu}_{\boldsymbol{B}}(x) & =n-\sum_{i=1}^{n} \boldsymbol{\mu}_{\boldsymbol{B} \Rightarrow \neg \boldsymbol{A}_{i}}(x), \\
\boldsymbol{\mu}_{\boldsymbol{B}}(x) & =\frac{\sum_{i=1}^{n} \boldsymbol{\mu}_{\neg \boldsymbol{A}_{i} \Rightarrow \boldsymbol{B}}(x)-1}{n-1} .
\end{aligned}
$$

\begin{tabular}{|c|c|c|c|c|c|c|c|}
\hline$\overbrace{\infty}$ & $\stackrel{\pi}{a}$ & $\stackrel{\infty}{\infty}_{\infty}^{\infty}$ & $\widehat{\oplus}$ & $R\left(\boldsymbol{A}_{\mathbf{1}} \Rightarrow \boldsymbol{B}\right)$ & $R\left(\boldsymbol{A}_{\mathbf{2}} \Rightarrow \boldsymbol{B}\right)$ & $R\left(\boldsymbol{A}_{\mathbf{3}} \Rightarrow \boldsymbol{B}\right)$ & $\sum_{i=1}^{n} R\left(\boldsymbol{A}_{\boldsymbol{i}} \Rightarrow \boldsymbol{B}\right)-n+1$ \\
\hline 1 & 0 & 0 & 1 & 1 & 1 & 1 & 1 \\
\hline 1 & 0 & 0 & 0 & 0 & 1 & 1 & $\mathbf{0}$ \\
\hline 0 & 1 & 0 & 1 & 1 & 1 & 1 & 1 \\
\hline 0 & 1 & 0 & 0 & 1 & 0 & 1 & 0 \\
\hline 0 & 0 & 1 & 1 & 1 & 1 & 1 & 1 \\
\hline 0 & 0 & 1 & $\mathbf{0}$ & 1 & 1 & 0 & 0 \\
\hline
\end{tabular}

\begin{tabular}{|c|c|c|c|c|c|c|c|}
\hline$\underset{\Psi}{\Psi}$ & $\underset{\longleftarrow}{\pi}$ & 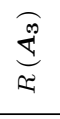 & $\underset{\Omega}{\underline{2}}$ & $R\left(\boldsymbol{B} \Rightarrow \boldsymbol{A}_{\mathbf{1}}\right)$ & $R\left(\boldsymbol{B} \Rightarrow \boldsymbol{A}_{\mathbf{2}}\right)$ & $R\left(\boldsymbol{B} \Rightarrow \boldsymbol{A}_{\mathbf{3}}\right)$ & $\frac{n-\sum_{i=1}^{n} R\left(\boldsymbol{B} \Rightarrow \boldsymbol{A}_{i}\right)}{n-1}$ \\
\hline 1 & 0 & 0 & 1 & 1 & 0 & 0 & 1 \\
\hline 1 & 0 & 0 & 0 & 1 & 1 & 1 & 0 \\
\hline 0 & 1 & 0 & 1 & 0 & 1 & 0 & 1 \\
\hline 0 & 1 & 0 & 0 & 1 & 1 & 1 & 0 \\
\hline 0 & 0 & 1 & 1 & 0 & 0 & 1 & 1 \\
\hline 0 & 0 & 1 & 0 & 1 & 1 & 1 & 0 \\
\hline
\end{tabular}

\section{Example}

Let us show that in standard two-valued logic, the relations (37) and (38) hold for four sentences $\boldsymbol{A}_{1}, \boldsymbol{A}_{2}, \boldsymbol{A}_{3}$, and $\boldsymbol{B}$ :

Tab. II Demonstration of the validity of formula (37).

Tab. III Demonstration of the validity of formula (38).

We see that the fourth and eighth columns of Tabs. II and III are the same. 


\subsection{Modus tollens}

Modus tollens is a logical rule that says: If $A \Rightarrow B$ holds and $B$ does not hold, then $A$ does not hold.

In floppy logic, we can derive these generalizations of modus tollens:

$$
\begin{aligned}
& R(\neg \boldsymbol{A})=R(\boldsymbol{A} \Rightarrow \boldsymbol{B})+R(\boldsymbol{A} \Rightarrow \neg \boldsymbol{B})-1, \\
& R(\neg \boldsymbol{A})=R(\boldsymbol{A} \Rightarrow \boldsymbol{B})+R(\neg \boldsymbol{B})-R(\boldsymbol{B} \Rightarrow \boldsymbol{A}), \\
& R(\neg \boldsymbol{A})=2-R(\neg \boldsymbol{A} \Rightarrow \boldsymbol{B})-R(\boldsymbol{B} \Rightarrow \boldsymbol{A}), \\
& R(\neg \boldsymbol{A})=R(\boldsymbol{B} \Rightarrow \neg \boldsymbol{A})+R(\boldsymbol{B})-R(\neg \boldsymbol{A} \Rightarrow \boldsymbol{B}) . \\
& \boldsymbol{\mu}_{\neg \boldsymbol{A}}(x)=\boldsymbol{\mu}_{\boldsymbol{A} \Rightarrow \boldsymbol{B}}(x)+\boldsymbol{\mu}_{\boldsymbol{A} \Rightarrow \neg \boldsymbol{B}}(x)-1, \\
& \boldsymbol{\mu}_{\neg \boldsymbol{A}}(x)=\boldsymbol{\mu}_{\boldsymbol{A} \Rightarrow \boldsymbol{B}}(x)+\boldsymbol{\mu}_{\neg \boldsymbol{B}}(x)-\boldsymbol{\mu}_{\boldsymbol{B} \Rightarrow \boldsymbol{A}}(x), \\
& \boldsymbol{\mu}_{\neg \boldsymbol{A}}(x)=2-\boldsymbol{\mu}_{\neg \boldsymbol{A} \Rightarrow \boldsymbol{B}}(x)-\boldsymbol{\mu}_{\boldsymbol{B} \Rightarrow \boldsymbol{A}}(x), \\
& \boldsymbol{\mu}_{\neg \boldsymbol{A}}(x)=\boldsymbol{\mu}_{\boldsymbol{B} \Rightarrow \neg \boldsymbol{A}}(x)+\boldsymbol{\mu}_{\boldsymbol{B}}(x)-\boldsymbol{\mu}_{\neg \boldsymbol{A} \Rightarrow \boldsymbol{B}}(x) .
\end{aligned}
$$

\subsection{Modus tollens for a finite partition of the sample space}

Let $\left\{\boldsymbol{B}_{i}\right\}$ be a finite set of $n$ pairwise disjoint floppy sets whose union is the entire sample space. Then modus tollens can be generalized:

$$
\begin{aligned}
& R(\neg \boldsymbol{A})=\frac{\sum_{i=1}^{n} R\left(\boldsymbol{A} \Rightarrow \boldsymbol{B}_{i}\right)-1}{n-1}, \\
& R(\neg \boldsymbol{A})=n-\sum_{i=1}^{n} R\left(\boldsymbol{B}_{i} \Rightarrow \boldsymbol{A}\right), \\
& R(\neg \boldsymbol{A})=\sum_{i=1}^{n} R\left(\boldsymbol{B}_{i} \Rightarrow \neg \boldsymbol{A}\right)-n+1, \\
& R(\neg \boldsymbol{A})=\frac{n-\sum_{i=1}^{n} R\left(\neg \boldsymbol{A} \Rightarrow \boldsymbol{B}_{i}\right)}{n-1}, \\
& \boldsymbol{\mu}_{\neg \boldsymbol{A}}(x)=\frac{\sum_{i=1}^{n} \boldsymbol{\mu}_{\boldsymbol{A} \Rightarrow \boldsymbol{B}_{i}}(x)-1}{n-1}, \\
& \boldsymbol{\mu}_{\neg \boldsymbol{A}}(x)=n-\sum_{i=1}^{n} \boldsymbol{\mu}_{\boldsymbol{B}_{i} \Rightarrow \boldsymbol{A}}(x), \\
& \boldsymbol{\mu}_{\neg \boldsymbol{A}}(x)=\sum_{i=1}^{n} \boldsymbol{\mu}_{\boldsymbol{B}_{i} \Rightarrow \neg \boldsymbol{A}}(x)-n+1, \\
& \boldsymbol{\mu}_{\neg \boldsymbol{A}}(x)=\frac{n-\sum_{i=1}^{n} \boldsymbol{\mu}_{\neg \boldsymbol{A} \Rightarrow \boldsymbol{B}_{i}}(x)}{n-1} .
\end{aligned}
$$




\subsection{Relations between implications}

Implications can be substituted according to the following rules:

$$
\begin{aligned}
& R(\boldsymbol{A} \Rightarrow \boldsymbol{B})=R(\boldsymbol{B} \Rightarrow \boldsymbol{A})-R(\boldsymbol{A})+R(\boldsymbol{B}), \\
& R(\boldsymbol{A} \Rightarrow \boldsymbol{B})=R(\boldsymbol{B})+1-R(\neg \boldsymbol{A} \Rightarrow \boldsymbol{B}), \\
& R(\boldsymbol{A} \Rightarrow \boldsymbol{B})=R(\neg \boldsymbol{A})+1-R(\boldsymbol{A} \Rightarrow \neg \boldsymbol{B}), \\
& \boldsymbol{\mu}_{\boldsymbol{A} \Rightarrow \boldsymbol{B}}(x)=\boldsymbol{\mu}_{\boldsymbol{B} \Rightarrow \boldsymbol{A}}(x)-\boldsymbol{\mu}_{\boldsymbol{A}}(x)+\boldsymbol{\mu}_{\boldsymbol{B}}(x), \\
& \boldsymbol{\mu}_{\boldsymbol{A} \Rightarrow \boldsymbol{B}}(x)=\boldsymbol{\mu}_{\boldsymbol{B}}(x)+1-\boldsymbol{\mu}_{\neg \boldsymbol{A} \Rightarrow \boldsymbol{B}}(x), \\
& \boldsymbol{\mu}_{\boldsymbol{A} \Rightarrow \boldsymbol{B}}(x)=\boldsymbol{\mu}_{\neg \boldsymbol{A}}(x)+1-\boldsymbol{\mu}_{\boldsymbol{A} \Rightarrow \neg \boldsymbol{B}}(x) . \\
& R(\boldsymbol{A} \Rightarrow \boldsymbol{B})+R(\boldsymbol{A} \Rightarrow \neg \boldsymbol{B})+R(\neg \boldsymbol{A} \Rightarrow \boldsymbol{B})+R(\neg \boldsymbol{A} \Rightarrow \neg \boldsymbol{B})=3, \\
& \boldsymbol{\mu}_{\boldsymbol{A} \Rightarrow \boldsymbol{B}}(x)+\boldsymbol{\mu}_{\boldsymbol{A} \Rightarrow \neg \boldsymbol{B}}(x)+\boldsymbol{\mu}_{\neg \boldsymbol{A} \Rightarrow \boldsymbol{B}}(x)+\boldsymbol{\mu}_{\neg \boldsymbol{A} \Rightarrow \neg \boldsymbol{B}}(x)=3 .
\end{aligned}
$$

\section{Conclusion}

Floppy logic is a new multi-valued logic that has significant links to fuzzy logic, the theory of probability, probability logic, and standard Boolean logic.

Its relationship to the theory of probability is already described in [8], where we prove that floppy logic is a model of Kolmogorov probability theory. This means that it is possible to use all the tools and findings of standard probability theory in floppy logic.

The relationship to fuzzy logic is given primarily by the fact that floppy logic also works with fuzzy sets and their membership functions. The main difference is due to floppy logic not describing phenomena directly with fuzzy sets but with sets of fuzzy sets [8].

In this article, we formulated and proved the isomorphism theorem. This has the following two consequences:

1) All statements which are equivalent in standard Boolean logic are also equivalent in floppy logic.

2) Floppy logic has all the properties of standard Boolean logic which can be formulated as an equivalence.

It is worth noting that fuzzy logic cannot possess these properties.

Like probabilistic logic, floppy logic works with probabilities of statements. In this article, we used the isomorphism theorem to derive the formula for floppy implication. This formula differs from Adams' Thesis in probabilistic logic. Therefore, floppy logic is not limited by Lewis' triviality result, which is a great advantage over probabilistic logic.

The isomorphism theorem also significantly clarified the relationship between floppy logic and standard Boolean logic. The two aforementioned consequences of 
the isomorphism theorem lead us to the notion that floppy logic is a multi-valued generalization of standard Boolean logic.

Another practical result of this article is the derivation of an entire range of inference rules which generalize modus ponens and modus tollens. These rules not only apply in floppy logic but also in standard Boolean logic if we replace the probabilities with truth values 0 and 1 .

\section{Appendix I - Explanation of Concepts}

\section{Boolean Algebra}

A Boolean algebra is a non-empty set $B$, together with two binary operations $\wedge$ and $\vee($ on $B)$, a unary operation ', and two distinguished elements 0 and 1 , satisfying the following axioms [2, p. 10]:

$$
\begin{array}{rlrl}
0^{\prime} & =1, & 1^{\prime} & =0, \\
p \wedge 0 & =0, & p \vee 1 & =1, \\
p \wedge 1 & =p, & p \vee 0 & =p, \\
p \wedge p^{\prime} & =0, & p \vee p^{\prime} & =1, \\
\left(p^{\prime}\right)^{\prime} & =p, & p \vee p & =p, \\
p \wedge p & =p, & (p \vee q)^{\prime} & =p^{\prime} \wedge q^{\prime}, \\
(p \wedge q)^{\prime} & =p^{\prime} \vee q^{\prime}, & p \vee q & =q \vee p, \\
p \wedge q & =q \wedge p, & p \vee(q \vee r) & =(p \vee q) \vee r, \\
p \wedge(q \wedge r) & =(p \wedge q) \wedge r, & p \vee(q \wedge r) & =(p \vee q) \wedge(p \vee r) . \\
p \wedge(q \vee r) & =(p \wedge q) \vee(p \wedge r), &
\end{array}
$$

These axioms are not independent. Often a smaller group of axioms is chosen.

Boolean algebra is often denoted as follows: $\left(B, \wedge, \vee,,^{\prime}, 0,1,=\right)$. Operations $\wedge$, $\vee$, and ' are often called meet, join, and complement. Elements 0 and 1 are called zero and one.

\section{Atom}

An atom $q$ of a Boolean algebra $\mathcal{B}$ is an element that meets these propositions [2, p. 117]:

$$
\begin{array}{ll}
\text { a. } & q \neq 0, \\
\text { b. } & \text { for every element } p \in \mathcal{B} \text {, either } p \wedge q=q \text { or } p \wedge q=0 \text {, but not both. }
\end{array}
$$

Example 1: Atoms of a Boolean algebra of sentences given by sentence $A_{1}, A_{2}$, $A_{3}, \ldots A_{n}$ are sentences $B_{1} \wedge B_{2} \wedge B_{3} \wedge \ldots \wedge B_{n}$ where either $B_{i}=A_{i}$ or $B_{i}=\neg A_{i}$.

Example 2: Atoms of a Boolean algebra of subsets of $\boldsymbol{S}$ are one-element subsets of $\boldsymbol{S}$. 


\section{Isomorhism}

Isomorphism $f$ of two Boolean algebras $\mathcal{B}_{1}$ and $\mathcal{B}_{2}$ is a bijection that preserves the operations meet, join, and complement. Thus:

$$
\begin{array}{ll}
\text { a. } & \forall p, q \in \mathcal{B}_{1}: f(p \wedge q)=f(p) \wedge f(q), \\
\text { b. } & \forall p, q \in \mathcal{B}_{1}: f(p \vee q)=f(p) \vee f(q), \\
\text { c. } & \forall p \in \mathcal{B}_{1}: f\left(p^{\prime}\right)=f(p)^{\prime} .
\end{array}
$$

\section{Appendix II - Proof of theorem 2}

The proof begins with the statement: "Every finite Boolean algebra $\mathcal{B}$ is isomorphic to the field $\mathcal{P}(n)$, or, equivalently, to the Boolean algebra $2^{n}$, for some non-negative integer $n$. In fact, $n$ is the number of atoms in $\mathcal{B} "[2$, p. 127].

$\varepsilon$ and $\delta$ are finite algebras, $\mathcal{P}(\boldsymbol{S})$ is the field $\mathcal{P}(n), n$ is the number of atoms in $\varepsilon$ and $\delta$. Therefore, $\varepsilon$ is isomorphic to $\delta$.

Thus, an isomorphism between $\varepsilon$ and $\delta$ exists. Let us find it:

Let $g$ be a binary relation over $E$ and a set $B$ of binary indices of length $n$.

The indices to the statements are as follows: If a sentence $V$ meets $V \wedge U_{k}=U_{k}$, then 1 is in the $k$-th place.

If $V$ meets $V \wedge U_{k}=\perp$, then 0 is in the $k$-th place. No other possibility exists because $U_{k}$ are atoms.

Let us show that $g$ is a bijection:

a. Each statement is assigned a binary index. Thus, $g$ is a left-total relation .

b. Each statement is assigned exactly one binary index.

Let us suppose that two equivalent sentences $V \equiv W$ are assigned two different binary indices. Let these indices be different in $k$-th place. It means that $V \wedge U_{k}=U_{k}$ and $W \wedge U_{k}=\perp$ (or vice versa).

$V$ and $W$ are equivalent and can thus be exchanged: $W \wedge U_{k}=U_{k}$. Thus, $U_{k}=\perp$, which is a contradiction since $U_{k}$ is an atom and atoms are non-zero elements.

Thus, $g$ is a left-total function.

c. Each binary index is assigned a statement. The statement $U_{i} \vee U_{j} \vee U_{k} \vee \ldots$ can be assigned to the binary index with 1 in the $i$-th, $j$-th, $k$-th, ...places.

Thus, $g$ is a surjection.

d. $E$ and $B$ are finite sets with the same number of elements. The number of elements is $2^{n}[2$, p. 82].

Thus, $g$ is a bijection.

Let $h$ be a binary relation $\mathcal{P}(\boldsymbol{S})$ to the set $B$ of binary indices of length $n$.

The indices are assigned to the floppy sets as follows:

If a floppy set $\boldsymbol{B}$ meets $\boldsymbol{B} \cap\left\{A_{k}\right\}=\left\{A_{k}\right\}$, then 1 is in the $k$-th place.

If $\boldsymbol{B} \cap\left\{A_{k}\right\}=\emptyset$, then 0 is in the $k$-th place. 
$h$ is also a bijection. It can be proven in the same way as bijection $g$. Thus $h^{-1} \circ g$ is a bijection. Let us show that $h^{-1} \circ g$ is an isomorphism:

a. Preservation of the binary operation meet:

Sentences $V$ and $W$ have the same binary indices as floppy sets $h^{-1} \circ g(V)$ and $h^{-1} \circ g(W)$.

$V \wedge W$ has a binary index where 1 is in the places where 1 is in both binary indices of $V$ and $W$ simultaneously.

$h^{-1} \circ g(V) \cap h^{-1} \circ g(W)$ has a binary index where 1 is in the places where 1 is in both binary indices of $h^{-1} \circ g(V)$ and $h^{-1} \circ g(W)$ simultaneously.

Thus, $V \wedge W$ and $h^{-1} \circ g(V) \cap h^{-1} \circ g(W)$ have the same binary index.

Thus: $\forall V, W \in E: h^{-1} \circ g(V \wedge W)=h^{-1} \circ g(V) \cap h^{-1} \circ g(W)$.

b. Preservation of binary operation join can be proved in the same way.

c. Preservation of unary operation complementation:

A binary index of sentence $\perp$ has 0 in all positions since $\forall k: \perp \wedge U_{k}=\perp$.

A binary index of sentence $\top$ has 1 in all positions since $\forall k: \top \wedge U_{k}=U_{k}$.

Using the axiom of Boolean Algebra: $V \wedge \neg V=\perp$. Thus, in the index of $\neg V, 1$ must not be in the positions where 1 is in the index of $V$.

Now, using the axiom: $V \vee \neg V=\top$. Thus, in the index of $\neg V, 1$ must be in all positions where 1 is not in the index of $V$.

Thus, the index of $\neg V$ has 1 exactly in these positions where $V$ has 0 .

Similarly, it can be shown that $\left(h^{-1} \circ g(V)\right)^{\prime}$ has 1 exactly in these positions where $h^{-1} \circ g(V)$ has 0 .

Therefore, $\neg V$ and $\left(h^{-1} \circ g(V)\right)^{\prime}$ have the same binary indices.

Thus: $\forall V \in E: h^{-1} \circ g(\neg V)=\left(h^{-1} \circ g(V)\right)^{\prime}$.

d. Preservation of neutral elements:

Sentence $\perp$, as well as empty floppy set $\emptyset$, has a binary index with all zeros. Thus:

$h^{-1} \circ g(\perp)=\emptyset$.

Similarly, it can be shown that $h^{-1} \circ g(\top)=\boldsymbol{S}$.

Bijection $h^{-1} \circ g$ preserves all operations, so it is an isomorphism.

Let us show that $h^{-1} \circ g$ meets all properties of relation $f$ :

Properties (7), (8), and (9) were proved above. Now, we prove property (6):

First, we apply: $U_{i} \wedge U_{j}=\perp$, if $i \neq j$. The second possibility for atoms $U_{i} \wedge U_{j}=U_{i}$ cannot apply since $U_{j}$ would not be an atom, which is a contradiction.

Thus, all atoms have only one 1 in their binary indices. Sentence $U_{k}$ has 1 in the $k$-th position as well as floppy set $\left\{A_{k}\right\}$.

Thus: $\forall k \in\{1,2,3, \ldots n\}: h^{-1} \circ g\left(U_{k}\right)=\left\{A_{k}\right\}$. 
Now, we show that $h^{-1} \circ g=f$.

First, we denote a set of all sentences whose binary indices contain no more than $k 1$ as $M_{k}$.

It was proved above that $h^{-1} \circ g\left(U_{k}\right)=f\left(U_{k}\right)$.

Now, we show that $h^{-1} \circ g(\perp)=f(\perp)$. It is simple, because $h^{-1} \circ g(\perp)=\emptyset$ and $f(\perp)=f(V \wedge \neg V)=f(V) \cap f(\neg V)=f(V) \cap f(V)^{\prime}=\emptyset$.

The equivalence $h^{-1} \circ g=f$ for all sentences from $M_{1}$ is thus proved.

All sentences from $M_{2}$ can be expressed as a disjunction of two sentences from $M_{1}$ :

$\forall Z \in M_{2}: \exists V, W \in M_{1}: Z=V \vee W$

Thus:

$$
\begin{aligned}
h^{-1} \circ g(Z) & =h^{-1} \circ g(V \vee W)= \\
& =h^{-1} \circ g(V) \cup h^{-1} \circ g(W)= \\
& =f(V) \cup f(W)=f(Z) .
\end{aligned}
$$

The equivalence $h^{-1} \circ g=f$ for all sentences from $M_{2}$ is thus proved.

This procedure can be repeated to prove equivalence $h^{-1} \circ g=f$ for $M_{3}$, $M_{4}, \ldots M_{n}$

Since $M_{n}=E$, the equivalence $h^{-1} \circ g=f$ is proved for all sentences from $E$.

Therefore, $f$ is an isomorphism.

It represents the first assertion of Theorem 2. The following assertions are implied immediately.

\section{References}

[1] ADAMS E.W. The logic of conditionals: An application of probability to deductive logic. Dordrecht: Springer Science \& Business Media, 1975. ISBN 9789048183432.

[2] GIVANT S., HALMOS P. Introduction to Boolean Algebras. New York: Springer Science+Business Media, 2009. ISBN 978-0-387-68436-9.

[3] KUKLOVA J, PŘIBYL O. Changeover from decision tree approach to fuzzy logic approach within highway management. Neural Network World. 2017, 27(2), pp. 181, doi: $10.14311 / \mathrm{NNW} .2017 .27 .008$.

[4] LEWIS D. Probabilities of conditionals and conditional probabilities. In: W.L. HARPER, R. STALNAKER, G. PEARCE, eds. Ifs. Dordrecht: Springer, 1976, pp. 129-147. ISBN 978-94-009-9117-0.

[5] ŁUKASIEWICZ J. O logice trójwartościowej (On three-valued logic). Ruch Filozoficzny. 1920, 6, pp. 170-171.

[6] NABOVATI H, HALEH H, VAHDANI B. Fuzzy multi-objective optimization algorithms for solving multi-mode automated guided vehicles by considering machine break time and artificial neural network. Neural Network World. 2018, 28(3), pp. 255-283, doi: 10.14311/NNW.2018.28.016.

[7] NAVARA M., OLŠÁK P. Základy fuzzy množin (Basics of Fuzzy Sets). Praha: Vydavatelství ČVUT, 2002. ISBN 80-01-02585-3. 
Provinský P.: Floppy logic as a generalization of standard Boolean logic

[8] PROVINSKÝ P. Floppy Logic - a Younger Sister of Fuzzy Logic. Neural Network World. 2017, vol. 27(issue 5), pp. 479-497, doi: 10 . 14311/NNW . 2017 . 27 . 025. ISSN 2336-4335.

[9] PROVINSKÝ P. Floppy Logic - Instructions for Use. Neural Network World. 2018, 28(5), pp. 473-494, doi: 10.14311/NNW. 2018.28.026. ISSN 2336-4335.

[10] STALNAKER R.C. Probability and conditionals. Philosophy of science. 1970, 37(1), pp. 64-80, doi: 10.1086/288280. ISSN 00318248.

[11] ZADEH L.A. Fuzzy sets. Information and Control. 1965, 8(3), pp. 338-353, doi: 10. 1016/S0019-9958(65)90241-X. ISSN 00199958. 
